FERMILAB-TM-1760

\title{
Energy and Energy Width Measurement in the FNAL Antiproton Accumulator
}

M. Church, S. Hsueh, P. Rapidis and S. Werkema

Fermi National Accelerator Laboratory

P.O. Box 500, Batavia, Illinois 60510

October 1991 


\section{Disclaimer}

This report was prepared as an account of work sponsored by an agency of the United States Government. Neither the United States Government nor any agency thereof, nor any of their employees, makes any warranty, express or implied, or assumes any legal liability or responsibility for the accuracy, completeness, or usefullness of any information, apparatus, product, or process disclosed, or represents that its use would not infringe privately owned rights. Reference herein to any specific commercial product, process, or service by trade name, trademark, manufacturer, or otherwise, does not necessarily constitute or imply its endorsement, recommendation, or favoring by the United States Government or any agency thereof. The views and opinions of authors expressed herein do not necessarily state or reflect those of the United States Government or any agency thereof. 


\title{
Energy and Energy Width Measurement in the FNAL Antiproton Accumulator
}

\author{
M. Church, S.Y. Hsueh, P.A. Rapidis, S. Werkema \\ Fermi National Accelerator Laboratory*
}

\section{Abstract}

The Fermilab Antiproton Accumulator has recently been used to produce Charmonium resonances (charm quark, anti-charm quark bound states) in protonantiproton annihilations using an internal $\mathrm{H}_{2}$ gas jet target. A measurement of the resonance mass and width may be obtained from a precise knowledge of the antiproton beam energy and energy spread. The beam energy is measured to an accuracy of 1 part in $10^{4}$ in the range $6.3 \mathrm{Gev}$ to $4.1 \mathrm{Gev}$ by measuring the orbit length and revolution frequency of the beam. The beam momentum spread is measured to an accuracy of $10 \%$ by messuring the beam frequency spread and the parameter $\eta=\left(P_{\text {beam }} / F_{\text {rev }}\right) \cdot\left(d F_{\text {rev }} / d P_{\text {beam }}\right)$. These two measurement techniques are described in this report.

\section{INTRODUCTION}

A goal of Fermilab exp. E760 is to measure the masses and widths of charmonium states in the region 2.9 to 3.8 Gev $/ c^{2}{ }^{[1]}$ These states are produced by decelerating a stack of $\bar{p}^{\prime} s$ from the stacking energy of $8.9 \mathrm{Gev}$ to an energy between 6.3 and $4.1 \mathrm{Gev}$ and then colliding this beam with en internal $\mathrm{H}_{2}$ gas jet target. While the $\mathrm{H}_{2}$ jet is on, the beam is stochastically cooled in 3 dimensions so that $\epsilon_{h,-} \sim 1 x \mathrm{~mm}-\mathrm{mrad}$ and $\Delta P / P \approx 10^{-4}$. The beam energy is scanned in small steps across the charmonium state and the production of charmonium is measured as a function of beam energy. The effective cross section is a convolution of the beam energy spectrum and a Breit-Wigner:

$$
\sigma\left(E_{c m}\right)=\int_{0}^{\infty} \sigma_{B W}(E) \cdot S\left(E-E_{c m}\right) d E
$$

where $S\left(E-E_{c m}\right)$ is the beam energy spectrum for beam at center of mass energy $E_{\mathrm{em}}$. The resonance parameters can be determined from the above excitation curve if the beam energy spectrum is known.

- Operated by the Univeratien Research Asmociation under contract with the U.S. Department of Energy

\section{BEAM WIDTH MEASUREMENT}

\section{A. Frequency Spectrum}

The beam momentum spectrum is obtained from the beam revolution frequency spectrum by

$$
\Delta p / p=\frac{1}{\eta} \cdot \Delta f / f
$$

where

$$
\eta=\frac{1}{\gamma^{2}}-\frac{1}{\gamma_{T}^{2}}
$$

The beam signal is detected with o coarial quarter wavelength pickup which resonates at $79 \mathrm{Mhz}$, and the Schottky noise from the pickup is analyzed with an HP8568B spectrum analyzer. For DC beam the number of $\bar{p}$ 's in a given frequency range is proportional to the Schottky power in that range. The frequency spectrum is recorded once every 3 minutes and the average of 20 to 80 of these spectra is used to determine the beam frequency characteristics. Fig. 1 shows a typical spectrum taken at the 127 th harmonic of the revolution frequency. The data is fit to an asymmetric Gaussian with $\sigma_{\text {low }} 20 \%$ larger than $\sigma_{\text {high }}$. The low energy tail is due to energy losses in collisions with electrons in the $H_{2}$ gas jet.

The major uncertainty in determining the beam momentum spectrum is from the uncertainty in $\eta$ in eq. (2). $\eta$ is calculated by 2 different methods and, in addition, it

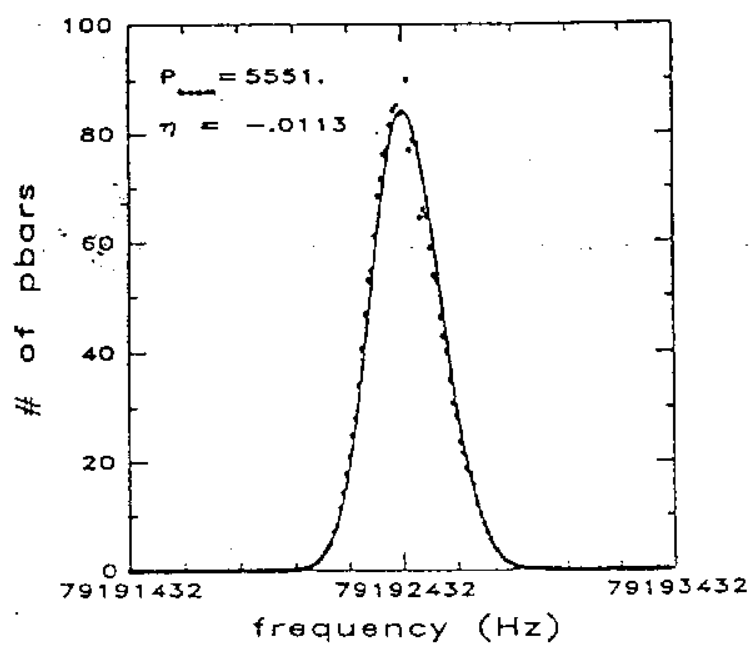

Figare 1: Frequency epectrom. Vertical scalc is arbitrary. 
can be determined at 2 different beam energies by a 3rd method denoted here as a "double scan."

\section{B. $\gamma_{T}$ Measurement}

The first method of measuring $\eta$ uses eq. (3). $\gamma_{T}$ is measured by changing the current in the main dipole bus and measuring the relative frequency change as a function of the relative magnetic field change:

$$
\gamma_{T}^{2}=\frac{d f / f}{d B / B} .
$$

This method has 2 drawbacks.

1) At small values of $\eta$ a small relative error in $\gamma_{T}$ will yield a large relative error in $\eta$ :

$$
\delta \eta / \eta=\left(\frac{1}{\left(\dot{\gamma}_{T} / \gamma\right)^{2}-1}\right) \cdot d \gamma_{T} / \gamma_{T}
$$

2) The NMR probe used to measure $B$ is within a magnet which shares the same bus with the other dipoles, but is not in the Accumulator ring. Since each dipole has a different current shunt, the measured $d B / B$ is not precisely the same as seen by the bearn.

\section{Synchrotron Frequency Measurement}

$\eta$ can be determined from a measurement of the synchrotron frequency of stationary bunched beam:

$$
|\eta|=\frac{2 \pi H f_{?}^{2} \beta^{2} E_{\text {beam }}}{f_{r f}^{2}\left(e V_{r f}\right)}
$$

where $f_{1}$ is the synchrotron frequency of small amplitude oscillations. In this method the beam is bunched at $\mathrm{H}=2$ and the frequency difference between the synchrotron sidebands in the beam Schottky signal is measured as a function of $\mathrm{rf}$ power. Since $E_{\text {beam }}$ and $f_{\mathrm{i} f}$ are known very precisely, the majoz uncertainty in this method comes from the calibration of the if voltage. The of gap voltage was measured directly with high voltage probes and an oscilloscope.

\section{Double Scan}

Fig. 2 is a graphical representation of this method. Data is collected at pt. 1 and then the beam is bunched and moved to pt. 2 (magnetic field constant) where a 2 nd set of data is collected. The magnetic field is then ramped to pt. 3 (energy constant) where data is again collected. This procedure continues through the remaining pts. The 2 sets of data-pts. $1,3,5, \ldots$ denoted as the central orbit, and pts. $2,4,6, \ldots$ denoted as the side orbit-are then plotted as 2 excitation curves as a function of $P_{\text {beam }}$ as measured on the central orbit. $\eta$ is then given by

$$
\eta=\frac{\Delta f_{\text {peak }} / f_{\text {peak }}}{\Delta P_{\text {peak }} / P_{\text {peak }}}
$$

where $\Delta f_{\text {peak }}$ and $\Delta P_{\text {peak }}$ are the difference in revolution frequency and momentum between the peaks of the two excitation curves. The accuracy of this method depends on how well the beam position can be controlled. Each point on the side orbit should have the same orbit length, and each point on the central orbit should have the same orbit length. To the extent that this is true, errors in the beam energy measurement will not enter into this determination of $\eta$. This is accomplished by careful use of the movable 4-8 Ghe momentum stochastic cooling pickup. The position of the beam at the A20 high dispersion region is determined by the position of the 48 pickup because the beam will tend to center itself under the pickup due to the action of stochastic cooling.

Table 1 lists the values of $\eta$ obtained by the 3 different measurement techniques. Except when the double scan technique is applicable, we use the synchrotron frequency method of determining $\eta$ and attach to it an uncertainty of $\pm 10 \%$.

Table 1

$\eta$ measurements

\begin{tabular}{|c|c|c|c|c|}
\hline $\begin{array}{c}P_{\text {beam }} \\
(\mathrm{Mev} / \mathrm{c})\end{array}$ & $\begin{array}{c}\text { Machine } \\
\text { lattice }\end{array}$ & $\begin{array}{c}\eta \\
\left(\gamma_{T}\right)\end{array}$ & $\begin{array}{c}\eta \\
\left(f_{\mathrm{s}}\right)\end{array}$ & $\begin{array}{c}\eta \\
\text { (double scan })\end{array}$ \\
\hline 4065 & 1 & .0186 & .0185 & .0182 \\
\hline 5550 & 1 & -.0046 & -.0053 & $\mathbf{x}$ \\
\hline 5550 & 2 & -.0104 & -.0113 & $x$ \\
\hline 5730 & 1 & -.0064 & -.0068 & $x$ \\
\hline 5730 & 2 & -.0122 & -.0135 & $x$ \\
\hline 6230 & 1 & -.0109 & -.0105 & -.0094 \\
\hline 6230 & 2 & -.0109 & -.0115 & $x$ \\
\hline
\end{tabular}

\section{ENERGY MEASUREMENT}

\section{A. Basic Technique}

The relativistic $\beta$ of the beam particle is given by

$$
\beta=f L / c
$$

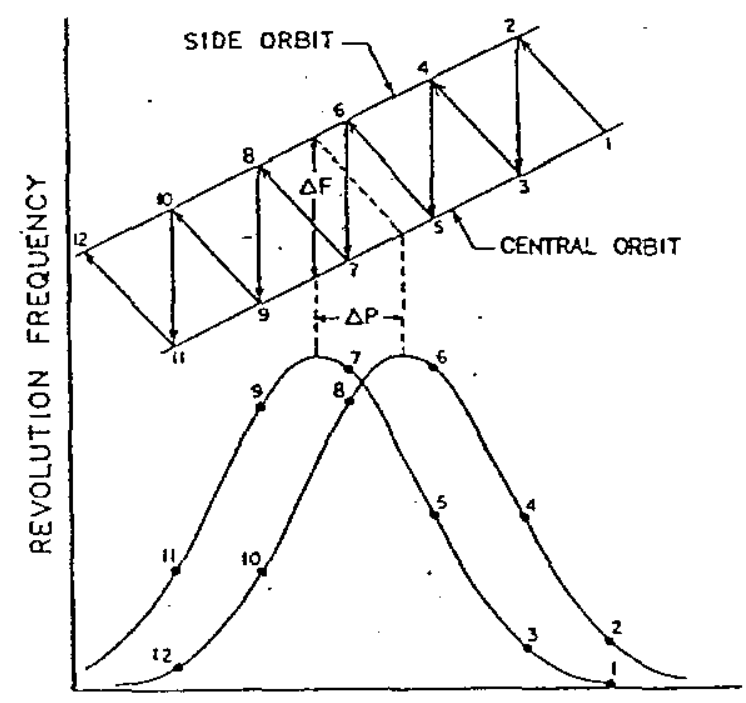

BEAM MOMENTUM

Figure 2: Graphical representation of double acan. Excitation curves are shom alro. 
where $f$ is the revolution frequency, $L$ is the orbit length $(\sim 474050 \mathrm{~mm})$, and $c$ is the speed of light. The error in the beam momentum is then given by

$$
\frac{d p}{p}=\gamma^{2} d \beta / \beta=\gamma^{2}\left(\frac{d f}{f}+\frac{d L}{L}\right) .
$$

$f$ is extracted from the frequency spectrum with an uncertainty of $2 / 10^{7}$ primarily due to the error in the determination of the peak of the spectrum. The spectrum snalyrer is calibrated to better than $1 / 10^{7}$ with an BP5335A frequency counter. The major source of error in $p$ comes from $d L$ which is calculated from the Beam Position Monitor (BPM) data, and is $\sim 1 \mathrm{~mm}$.

\section{B. Orbit Length Calculation}

The orbit length is given by

$$
L=L_{\text {ref }}+\delta L
$$

where $L_{\text {res }}$ is the known orbit length of a reference orbit obtained from an energy scan of an accurately measured chsrmonium state. The uncertainty in $L_{r e f}$ is determined by the uncertainty in the mass of the resonance:

$$
\delta L_{\text {ref }}=\frac{L_{r e f} \cdot M_{\Psi^{\prime}} \cdot \delta M_{\Psi^{\prime}}}{p^{2} \cdot \gamma}=.7 \mathrm{~mm}
$$

The length along an arbitrary path is given by

$$
L=\int\left[\left(\frac{d x}{d s}\right)^{2}+\left(1-\frac{x}{r}\right)^{2}\right]^{\frac{2}{2}} d s
$$

where the integral is over a reference curve $s, z$ is the lateral displacement from the reference curve, and $r$ is the local radius of curvature. In our case $\frac{z}{r}$ and $\frac{d x}{d q}$ are $\ll 1$ and eq. (12) can be reduced to

$$
L=\int d s+\int \frac{x}{r} d s=L_{r e f}+\sum_{i=1}^{30} \frac{x_{i}}{r_{i}} l_{d i p o l e}
$$

where the sum is over the 30 main dipoles in the Accumulator. The contributions from other magnets is negligible.

In the simplest approximation, the $x_{i}$ at the center of the dipoles can be calculated from the orbit difference measurements $\left(\triangle B P M_{j}\right)$ at the nearest BPMs by extrapolating through the quadrupoles using the thin lens approximation. In this case

$$
\delta L=\sum_{j=1}^{48} C_{j} \cdot \triangle B P M_{j}
$$

where $\triangle B P M_{j}$ ranges from 0 to $\pm 5 \mathrm{~mm}$.

This estimate of $\delta L$ does not account for a possible difference in curvature between the reference orbit and current orbit. To correct this, a more generally applicable technique has been developed. The deviation from the reference orbit is treated as a superposition of dipole kicks from all of the horizontally bending dipole elements in the Accumulator plus a $\Delta p / p$ error:

$z_{j}(\vec{\theta}, \Delta p / p)=\frac{\sqrt{\beta_{j}}}{2 \sin \pi \nu_{z}} \sum_{i=1}^{N_{2}} \sqrt{\beta_{i}} \theta_{i} \cos \left(\pi \nu_{z}-\Delta \phi_{i j}\right)+D_{j} \frac{\Delta p}{p}$

where the sum extends over all dipole elements in the Accumulator. $\vec{\theta}$ and $\Delta p / p$ in eq. (15) are determined from a least squares fit to the BPM difference measurements subject to the constraint that the net $\Delta p / p$ effect of the dipoles is zero. The resulting $\vec{\theta}$ and $\Delta p / p$ are then used to calculate the $\boldsymbol{x}_{\boldsymbol{i}}$.

\section{Beam Position Monitors}

Most of the error in the orbit length messurement comes from the BPMs. The orbit length is calculated from a large number of closed orbit measurements averaged together, and thus the error is dominated by systematics. Drift of electrical offsets, nonlinearities in the electronics, miscalibration, and a finite-sized LSB in the ADC all contribute a total estimated error in $\delta L$ of $\sim 1 \mathrm{~mm}$. During the double scan, any measured orbit length deviation must come from either variations in the dipole power supply regulation or systematic BPM errors. The orbit deviation at location $i$ from $N$ random dipole kicks around the ring is given by

$$
<\delta x_{i}^{2}>^{\frac{1}{5}}=\frac{\left(\beta_{i}<\beta>\right)^{\frac{1}{2}}}{2 \sqrt{2} \sin \pi \nu} N^{\frac{1}{2}}(\delta \theta)_{r m a} .
$$

Using $(\delta \theta)_{r m s} / \theta=1.4 \times 10^{-5}$ gives $d L \approx .17 \mathrm{~mm}$ from dipole regulation and ramps. The observed orbit deviations during the double scan were $\sim 1 \mathrm{~mm}$, and therefore we conclude this comes almost entirely from the BPMs.

Several problems with the BPMs have recently been corrected and it is believed that the systematic error in $\delta L$ can be reduced to $\sim .25 \mathrm{~mm}$.

\section{CONCLUSIONS}

The momentum width of stochastically cooled coasting beam in the Accumulator can be measured to an accuracy of $10 \%$, and several different measurement techniques provide independent checks on this. Beam energy can be measured to an accuracy estimated to be $1 / 10^{4}$. Further reduction in this error is expected with a more exact orbit length calculation and an improved BPM system.

\section{REFERENCES}

[1] V. Bharadwaj et al., "Operation of the Fermilab Accumulator for Medium Energy Proton-Antiproton Physics," in Proceedings of the European Particle Accelerator Conference, Nice, France, June 1990, pp 617-619. 